\title{
A Real-World Evaluation of the Long-Term Safety and Efficacy of Infliximab in the Treatment Moderate- to-Severe Psoriasis
}

\author{
Emily K. Haque (D) - Aaminah Azhar · John Corbett · Jillian Frieder (D) • \\ Xuan Wang $\cdot$ Alan Menter
}

Received: June 17, 2020 / Published online: August 20, 2020

(C) The Author(s) 2020

\begin{abstract}
Introduction: Psoriasis is a chronic immunemediated inflammatory skin disease that occurs in $2.5-3.5 \%$ of the general population. Infliximab (INF), a TNF- $\alpha$ inhibitor biologic agent, is a long-standing efficacious treatment for psoriasis; however, not all patients sustain a long-term response (LTR) because of a number of factors including antibody production. There is a paucity of studies assessing infliximab efficacy over a period $\geq 5$ years.
\end{abstract}

Digital Features To view digital features for this article go to https://doi.org/10.6084/m9.figshare.12794432.

Electronic supplementary material The online version of this article (https://doi.org/10.1007/s13555020-00436-1) contains supplementary material, which is available to authorized users.

E. K. Haque · A. Azhar

Texas A\&M Health Science Center, College of

Medicine, Dallas, TX, USAE. K. Haque

e-mail: Emily.Haque@tamu.edu

J. Corbett

Paul L Foster School of Medicine, Texas Tech

University Health Science Center, El Paso, TX, USA

J. Frieder $(\bowtie) \cdot$ A. Menter

Division of Dermatology, Baylor University Medical

Center, Dallas, TX, USA

e-mail: jhfrieder@gmail.com

X. Wang

Baylor Research Institute, Baylor University Medical

Center, Dallas, TX, USA
Methods: A retrospective cohort chart review of our clinic patients who had undergone $\geq 5$ years of treatment with infliximab for chronic plaque psoriasis was performed. The following variables were recorded and analyzed with the Fisher exact test: age, sex, body mass index ([BMI]; normal weight [NW], overweight $[\mathrm{OW}]$, obese $[\mathrm{OB}])$, changes in infliximab strength (dose or frequency), concomitant systemic therapy, and side effects. Clinical improvement was assessed by comparing the total body surface area (tBSA) affected by psoriasis before and after treatment.

Results: There was a significant difference in likelihood of achieving LTR between the NW, OW and OB groups $(p=0.044)$. Non-normalweight patients $(\mathrm{OW}+\mathrm{OB})$ were significantly more likely to achieve and sustain LTR than NW patients (OR 9.07, $p=0.020$ ). There were no other significant associations for the other evaluated variables.

Limitations: Patients who began treatment with infliximab before 2009 (prior to the use of the clinic's electronic medical record) were excluded. The Psoriasis Area and Severity Index (PASI) was not available for this study.

Conclusion: Surprisingly, patients who are overweight or obese are more likely to obtain long-term clinical benefit in their psoriasis symptoms with infliximab therapy than patients who are normal weight. 
Keywords: Antibodies to infliximab; Infliximab; Long-term response; Methotrexate; Obesity; Plaque psoriasis; Weight-based dose

\section{Key Summary Points}

\section{Why carry out this study?}

While the long-term safety of infliximab, a TNF- $\alpha$ inhibitor, has been well studied, there is a paucity of studies assessing longterm ( $\geq 5$ years) efficacy of infliximab

Psoriasis patients being treated with infliximab have frequently been reported to lose treatment response over time, and identifying potential factors associated with loss of response have not been well characterized

This retrospective study was conducted to identify variables associated with a sustained long-term response (LTR) in patients being treated for chronic moderate-to-severe plaque psoriasis with infliximab for at least 5 years

\section{What was learned from the study?}

In our 47-patient cohort, patients who were overweight or obese (BMI $\geq 25$ ) were more likely to achieve LTR than patients who were normal weight $(\mathrm{BMI}<25)$

These findings can guide clinicians when choosing optimal biologic therapy particularly in overweight and obese patients and emphasize the need for a thorough understanding of the weightbased mechanism of infliximab

\section{INTRODUCTION}

Psoriasis is a chronic, immune-mediated inflammatory skin disease that affects approximately $2.5-3.5 \%$ of the general population [1]. Psoriasis most commonly presents with welldemarcated, erythematous, scaly plaques and can lead to significant psychosocial stress $[2,3]$. Moreover, a state of chronic systemic inflammation links psoriasis with a number of comorbid conditions, including psoriatic arthritis, cardiovascular disease, metabolic syndrome (i.e., obesity, hypertension, and hyperlipidemia), autoimmune conditions (i.e., inflammatory bowel disease, alopecia areata, and vitiligo), malignancies (i.e., lymphoma), and psychiatric disorders (i.e., depression, anxiety, and suicidal ideation) [4-10]. Thus, psoriasis has a tremendous disease burden and can have a major impact on patients' quality of life. Due to the chronicity of the disease, treatment must provide not only an early and rapid response, but also long-term maintenance of efficacy.

While there is currently no cure for psoriasis, a relatively recent surge in new biologic agents (anti-IL-17 and IL-23 agents) approved for the treatment of moderate-to-severe plaque psoriasis offers patients the opportunity for clear or nearly clear skin, and thus improved quality of life [11]. With many psoriasis patients often hesitant to initiate treatment with newer agents that lack long-term safety data, infliximab's (INF) positive long-term safety profile offers reassurance and comfort despite the need for infusions. Moreover, analysis of patients with at least 5 years of INF therapy can identify positive and negative predictors for a positive outcome or sustained treatment response. These data will hopefully allow clinicians to identify patients who are optimal candidates for prolonged INF therapy.

Infliximab (Remicade ${ }^{\circledR}$, Johnson \& Johnson, 2006) was approved by the Federal Drug Administration (FDA) in 2006 for the treatment of moderate-to-severe plaque psoriasis. It is a chimeric IgGk monoclonal antibody composed of both human and murine regions that functions by neutralizing the biologic activity of $\mathrm{TNF} \alpha$ through inhibition of both soluble and transmembrane forms from binding to the receptor. Unlike other anti-TNF $\alpha$ biologic agents, INF is administered via intravenous infusion with a weight-based dosing, with an initial recommended dosage for plaque psoriasis of $5 \mathrm{mg} / \mathrm{kg}$ at weeks 0,2 , and 6 , then every 8 weeks [12]. Changes in INF dosage and/or 
frequency can be implemented if inadequate or significant loss of response occurs.

The efficacy and safety of treatment with INF for moderate-to-severe plaque psoriasis has been well studied [13-17]. However, there are limited data reporting the efficacy of INF after long periods of uninterrupted treatment beyond 1 year. Additionally, given that INF is a chimeric monoclonal antibody with a murine component, immunogenicity with the development of antidrug antibodies (ADA), or specifically antibodies to infliximab (ATIs), is an important consideration. These ATIs may reduce INF therapeutic efficacy and increase the risk of infusion reactions.

Clinically, adjustments in dosage and infusion frequency, as well as concomitant use of systemic immunosuppressive therapies (i.e., methotrexate), may assist in prolonging INF efficacy by either reducing or preventing ATIs. Additionally, patient demographics [i.e., age, race, and body mass index (BMI)] have been shown to have an impact on treatment longevity. Therefore, to evaluate the long-term response (LTR) of INF in patients with chronic moderate-to-severe psoriasis, a retrospective analysis on patients receiving continuous therapy for $\geq 5$ years in our clinic was completed. We hypothesize that of the investigated patient demographics, higher BMI will negatively correlate with LTR.

\section{METHODS}

\section{Study Design}

A retrospective chart review was performed on psoriasis patients who were treated in our psoriasis specialty dermatology clinic in Dallas, Texas, between 2009 and 2019. The primary purpose of the study is to identify positive and negative associations with a sustained treatment response in psoriasis patients receiving long-term treatment with INF for $\geq 5$ years.

\section{Data Collection}

All data were collected through the Athena electronic medical record (EMR) software. Medical records of patients with the International Statistical Classification of Diseases and Related Health Problems (ICD-10-CM) code of psoriasis (L-40.0, psoriasis vulgaris; L-40.1, generalized pustular psoriasis; L-40.2, acrodermatitis continua; L-40.3, pustulosis palmaris et plantaris; L-40.4, guttate psoriasis; L-40.8, other psoriasis; L-40.9, psoriasis unspecified) who received INF therapy during 1 January 2000 to 31 August 2019 were reviewed.

The data collected were comprised of patient demographics (age, sex, BMI), disease severity (TBSA at baseline and at 5 years of treatment), treatment modifications [total duration, dose or frequency (strength) increase], adjuvant methotrexate (MTX) or cyclosporine (CYA) use, response to treatment (\% improvement of TBSA), and side effects. Age was recorded as the patient's age at the time of treatment initiation, and patients were grouped as either $\geq 45$ or $<45$ years old. Sex was indicated by either male $(\mathrm{M})$ or female $(\mathrm{F})$. The patients were categorized into three groups according to their baseline BMI. Patients with a baseline BMI between 18.5 and 24.9 were grouped as normal weight (NW); patients with a baseline BMI between 25.0 and 29.99 were grouped as overweight (OW); patients with a baseline BMI $>30$ were grouped as obese (OB). Most patients had a quantitative value of TBSA in the EMR, but about a third of the patients did not have TBSA documented. For these patients, TBSA was estimated with the Wallace Rule of Nines and the physical examination describing the areas affected. The estimated TBSA was noted as (E), and the percent improvement of TBSA was reported with a numerical value. Duration of treatment was established by the time elapsed from the first date of infusion to the final or most recent date of infusion. A strength increase was given a binary value of yes $(\mathrm{Y})$ or no $(\mathrm{N})$, indicating whether the patient received either an increase in dose or increase in infusion frequency at any point in time during treatment. Patients were given a $\mathrm{Y}$ if they were either continuing previously prescribed MTX or CYA 
treatment or were newly prescribed MTX or CYA during the INF treatment period. If side effects were present, a short description was noted in Table S1.

\section{Patients}

We identified 438 patients who had ever been prescribed INF for treatment of psoriasis. Of this population, 305 patients had a treatment duration $<5$ years and 133 had a treatment duration $\geq 5$ years. Additionally, $75 / 133$ of the qualifying patients had initiated INF prior to the transition from paper charts to EMR in March 2009 and were excluded to allow for a more uniform and efficient analysis. Detailed chart review was performed on the remaining 58 patients and led to exclusion of 10 patients due to incomplete medical records, non-consecutive years of treatment, loss to follow-up, and inconsistent treatment defined as no infusion received for at least two consecutive intervals determined by the prescribed frequency at any point in the treatment duration (e.g., prescribed infusion frequency is q8 weeks; patient goes without infusion for at least 16 weeks [\# weeks without infusion/infusion frequency $\geq 2$ infusions] and therefore is excluded from this study.) Lastly, one pediatric patient was excluded. Data of 47 patients treated for psoriasis with INF for $\geq 5$ years from our specialty clinic were included in the final data set of the study (Table 1).

\section{Statistical Analyses}

The primary outcome, long-term response (LTR), was defined as a $\geq 90 \%$ improvement in tBSA at the end of the 5-year period compared to baseline. No LTR was defined as $<90 \%$ improvement in tBSA. Utilizing a cutoff of $\geq 75 \%$ for LTR would have resulted in an unbalanced binary outcome with $46 / 47$ patients meeting LTR. Patient BMI was dichotomized as a binary variable with the BMI cutoff of 25 .

Statistical analyses were performed using the Fisher exact test towards the primary outcome, LTR versus each of the five binary variables: age, sex, BMI group, strength increase $(\mathrm{Y} / \mathrm{N})$, and concomitant therapy $(\mathrm{Y} / \mathrm{N})$. Odds ratio (OR) and $p$ values are reported by the Fisher exact test.

\section{Compliance with Ethics Guidelines}

This retrospective analytic study was approved by the Baylor Scott \& White Research Institute's Institutional Review Board. All procedures performed in studies involving human participants were in accordance with the institutional research committee and with the 1964 Helsinki Declaration and its later amendments or comparable ethical standards. Informed consent was obtained from all individual participants included in the study.

\section{RESULTS}

The patient's quantitative (Table 2) and qualitative (Table 3) data were summarized. The BMI of the 47-patient sample ranged from 20.5 to 49.4. Out of 47 total patients, $25(52 \%)$ were obese (OB), 14 (30\%) were overweight (OW), 8 $(5.1 \%)$ were normal weight (NW), and one patient's BMI could not be calculated because the height was not documented. The mean (SD) BMIs for the NW, OW, and OB groups were 21.6 (1.0), 27.5 (1.2), and 37.8 (5.4), respectively. The mean (SD) duration of treatment for the NW, $\mathrm{OW}$, and $\mathrm{OB}$ groups were 6.56 (1.37), 6.54 (1.15), and 7.22 (1.55) years, respectively. The association between the variables and LTR were analyzed via Fisher exact test, and the corresponding odds ratio (OR) and $p$ values are indicated in Table 4. Statistical analysis is presented in Tables 5 and 6 . Of the variables collected and tested, there was a significant difference in the likelihood of achieving LTR among the NW, OW, and OB groups $(p=0.044)$. Additionally, NW was compared to non-NW $(\mathrm{OW}+\mathrm{OB})$ and was found to have a significant OR of $9.07[1.55,53.07](p=0.020)$.

\section{DISCUSSION}

INF has a rapid treatment response time demonstrated by a clinically meaningful benefit 


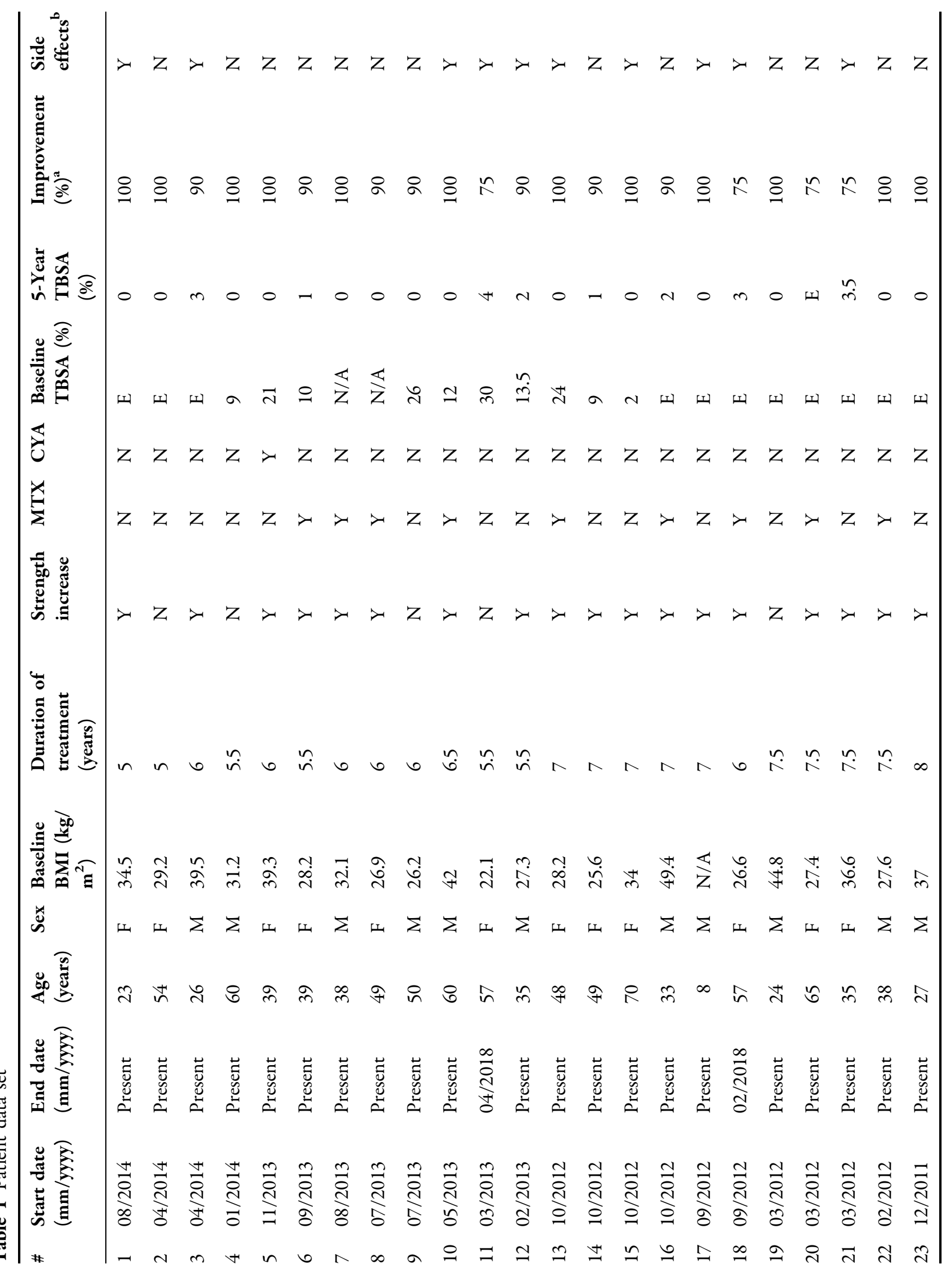




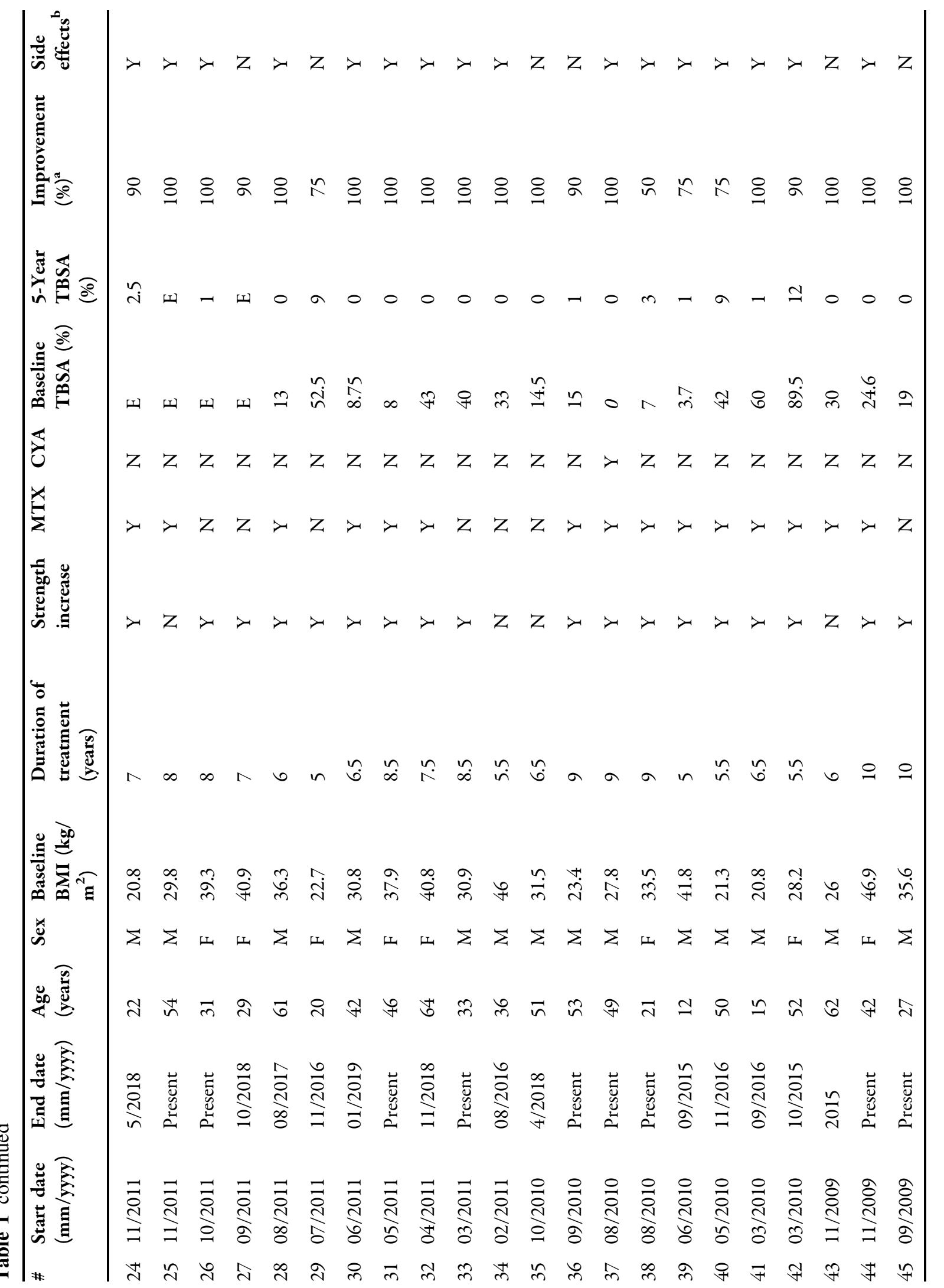




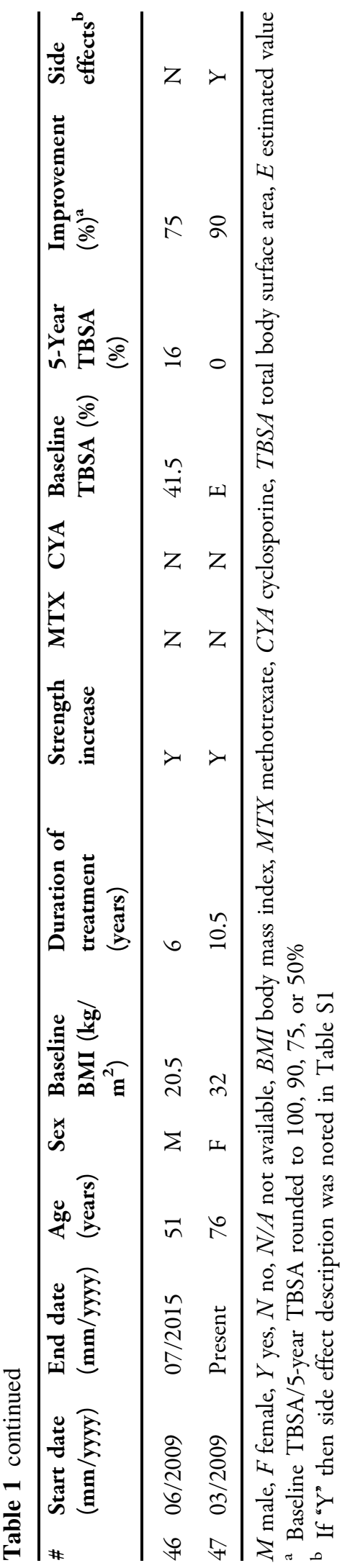

after 3.5 weeks ( 2 infusions), shorter than other biologics [18]. Because INF targets TNF- $\alpha$, an integral mediator in the underlying pathogenesis of psoriasis, it not only clears the cutaneous symptoms, but also has a significant effect on improving psoriatic arthritis symptoms [19]. INF is administered as an infusion continuously over a 90-120-min period (at constant intervals regardless of symptom severity) or rarely intermittently (only given when significant loss of response has occurred). Menter et al. found that sustained response was attained more often in patients undergoing a continuous regimen than an intermittent regimen [20].

The average BMI of psoriasis patients is reported to be higher than the BMI of the general population [21]. One meta-analysis conducted on 35 studies exploring the relationship between BMI and psoriasis reported that on average, psoriasis patients have a BMI $1.26 \mathrm{~kg} /$ $\mathrm{m}^{2}$ greater than adults and $1.55 \mathrm{~kg} / \mathrm{m}^{2}$ greater than children (5-18 years old) without psoriasis [22]. Among the five factors analyzed, only obesity was found to have a significant positive association with LTR. This finding goes against our hypothesis, as numerous studies have indicated that higher BMI is associated with an inferior treatment response to anti-TNF-alpha inhibitors, including INF, for a variety of inflammatory conditions [23]. This is related to obesity being associated with a constant state of chronic, low-grade inflammation [24]. Additionally, obesity can modify the pharmacokinetics of INF, leading to increased drug clearance and thus a shorter half-life and lower trough concentration levels [25]. An exception to this trend was seen in a pooled analysis of 1207 patients with inflammatory bowel disease (IBD), which revealed that obesity was not associated with an inferior response to INF. This may be related to differences in visceral and mesenteric fat distribution and its influence in IBD patients [26]; this cannot be applied to our psoriasis cohort. One potential physiologic explanation for our finding is that obesity can lead to an overall immunosuppressive state, with studies showing that increased adiposity promotes a reduced response to vaccinations and infections. Adipocytes produce leptin, a proinflammatory hormone, that may interfere 
Table 2 Summary of quantitative data

\begin{tabular}{llll}
\hline & Overall $(\boldsymbol{n}=\mathbf{4 7})$ & No LTR $(\boldsymbol{n}=\mathbf{9})$ & LTR $(\boldsymbol{n}=\mathbf{3 8})$ \\
\hline Age, years & $42.06(15.84)$ & $40.89(19.32)$ & $42.5(15.54)$ \\
Mean (SD) & & & \\
BMI, $\mathrm{kg} / \mathrm{m}^{2}$ & $32.0(7.6)$ & $28.1(7.6)$ & $33.2(7.3)$ \\
Mean (SD) & & & \\
Duration of treatment, years & $6.87(1.42)$ & $6.33(1.37)$ & $7.01(1.41)$ \\
Mean (SD) & &
\end{tabular}

LTR long-term response, BMI body mass index

with mounting an adequate adaptive immune response necessary for antigen-specific antibodies [27]. Perhaps this can also diminish the ability to make ATIs, leading to greater LTR [28]. Alternatively, these results could have been related to our small sample size and statistical methods. With only nine non-LRTs, our analysis capability was constrained to univariate analysis. Perhaps with a larger non-LRT cohort our results may have been different. The average BMI of psoriasis patients is reported to be higher than that of the general population (in the 30 range) [21]. INF is one of the only direct weightbased psoriasis therapies available. Therefore, the strength of INF treatment can be titrated according to weight to achieve maximum response.

\section{Patient Demographics: Age, Sex, and BMI}

There was no statistically significant difference between age $\geq 45$ and age $<45$ years or male vs. female response rates (Table 3 ). Of the patients in this study, 25/47 were obese with BMI ranging from 30.8 to 49.4 with a mean of 37.9. Obesity is a major factor that correlates with a positive likelihood of the LTR. Although INF is one of the very few psoriasis drugs titrated according to weight, the various comorbidities and proinflammatory effects associated with obesity may negate increases in INF dosing and/ or frequency, thus preventing optimal therapeutic outcomes. Therefore, the positive effects of INF treatment may be masked by the numerous variables associated with obesity. Højgaard et al. suggest that obesity is associated with higher disease activity and seemed to diminish response and adherence to TNF- $\alpha$ inhibitors in psoriatic arthritis [29].

\section{Total Duration of Treatment}

The mean (SD) duration of treatment for the NW, OW, and OB groups were 6.56 (1.37), 6.54 (1.15), and 7.22 (1.55) years, respectively. OB patients had a longer treatment duration, but it cannot be assumed this is only due to the good response with INF. For example, if a NW patient does not show adequate clinical benefit with INF, treatment would likely be switched to a different biologic, even with minor flares, as there are multiple alternative options now available. Conversely, OB patients may have already failed several biologic agents, so INF therapy is continued because of potentially fewer alternative agents available. Due to INF being one of the only weight-based medications for psoriasis, the best and only option for $\mathrm{OB}$ patients may have been to continue therapy and simply increase the dose $(\mathrm{mg} / \mathrm{kg})$ of treatment or shorten the 8-week interval between infusions even down to q4 weeks.

There was no significant difference in the mean duration of treatment between the LTR years and no LTR groups (Table 2). A larger population size is needed to identify any difference. ATIs may play a role in the duration of treatment needed to achieve LTR. 
Table 3 Summary of qualitative data

\begin{tabular}{|c|c|c|c|c|}
\hline & \multicolumn{2}{|c|}{ No LTR $(n=9)$} & \multicolumn{2}{|c|}{ LTR $(n=38)$} \\
\hline & Count & $\%$ & Count & $\%$ \\
\hline \multicolumn{5}{|l|}{ Age } \\
\hline$<45$ years & 4 & 56 & 20 & 47 \\
\hline$\geq 45$ years & 5 & 44 & 18 & 53 \\
\hline \multicolumn{5}{|l|}{ Sex } \\
\hline Male & 3 & 33 & 23 & 61 \\
\hline Female & 6 & 67 & 15 & 39 \\
\hline \multicolumn{5}{|l|}{ BMI } \\
\hline $\mathrm{NW}$ & 4 & 44 & 3 & 8 \\
\hline OW & 2 & 22 & 12 & 32 \\
\hline $\mathrm{OB}$ & 3 & 33 & 22 & 58 \\
\hline No BMI & 0 & 0 & 1 & 3 \\
\hline \multicolumn{5}{|c|}{ Strength increase } \\
\hline No & 1 & 11 & 8 & 21 \\
\hline Yes & 8 & 89 & 30 & 79 \\
\hline \multicolumn{5}{|c|}{ Concomitant therapy } \\
\hline Monotherapy & 4 & 44 & 18 & 47 \\
\hline MTX $^{a}$ & 2 & 22 & 19 & 50 \\
\hline $\mathrm{CYA}^{\mathrm{a}}$ & 0 & 0 & 2 & 5 \\
\hline \multicolumn{5}{|l|}{ Side effects } \\
\hline No & 3 & 33 & 17 & 45 \\
\hline Yes & 6 & 67 & 21 & 55 \\
\hline
\end{tabular}

$B M I$ body mass index, $N W$ normal weight, $O W$ overweight, $O B$ obese, $M T X$ methotrexate, $C Y A$ cyclosporine

a One patient used both MTX and CYA

\section{Strength (Dose or Frequency) Increase}

In the LTR group, 79\% (30/38) of patients required a dose or frequency increase, whereas $89 \%(8 / 9)$ of the patients without LTR required a dose or frequency increase (OR 0.47, $p=0.667$ ) (Table 4). Menter et al. reported higher PASI 75 response rates $(54.5 \%$ vs. $43.8 \%)$ for psoriasis patients randomized to INF $5 \mathrm{mg}$ / $\mathrm{kg}$ vs. $3 \mathrm{mg} / \mathrm{kg}$ Q8 weeks through week 50. Of note, PASI 75 response rates were higher for patients maintained on continuous (Q8 weeks) dosing vs. as-needed, regardless of dose $(3 \mathrm{mg} /$ $\mathrm{kg}$ or $5 \mathrm{mg} / \mathrm{kg}$ ). Importantly, there were no additional safety concerns for the $5 \mathrm{mg} / \mathrm{kg}$ dose compared to the $3 \mathrm{mg} / \mathrm{kg}$ dose [20]. This study demonstrated that a higher dose with scheduled maintenance therapy may provide better shortand long-term outcomes. Our findings indicate that patients requiring a higher dose over a longer period of time may be negatively associated with LTR. 
Table 4 Summary of statistical analysis

\begin{tabular}{|c|c|c|c|c|c|}
\hline & No LTR $(n=9)$ & $\operatorname{LTR}(n=38)$ & OR [95\% CI] & $p$ value & Conclusion \\
\hline Age & & & $0.72[0.17,3.10]$ & 0.66 & Age group did not significantly \\
\hline$<45$ years & 4 & 20 & & & affect LTR outcome \\
\hline$\geq 45$ years & 5 & 18 & & & \\
\hline Sex & & & $0.33[0.07,1.51]$ & 0.15 & Sex did not significantly affect \\
\hline Male & 3 & 23 & & & LTR outcome \\
\hline Female & 6 & 15 & & & \\
\hline BMI group & & & & & BMI group did significantly \\
\hline NW & 4 & 3 & & & affect LTR outcome \\
\hline OW & 2 & 12 & $8.00[0.96,66.46]$ & 0.05 & \\
\hline $\mathrm{OB}$ & 3 & 22 & $9.78[1.43,66.86]$ & 0.02 & \\
\hline No BMI & 0 & 1 & & & \\
\hline $\mathrm{BMI} \geq 25$ & & & $9.07[1.55,53.07]$ & $0.015^{*}$ & Patients with a BMI $\geq 25$ \\
\hline NW & 4 & 3 & & & were significantly more likely \\
\hline Non-NW & 5 & 34 & & & $\begin{array}{l}\text { to achieve LTR than patients } \\
\text { with a BMI }<24.9\end{array}$ \\
\hline Strength increase & & & $0.47[0.05,4.32]$ & 0.50 & Strength increase did not \\
\hline No & 1 & 8 & & & significantly affect LTR \\
\hline Yes & 8 & 30 & & & outcome \\
\hline Concomitant therapy & & & $0.90[0.21,3.83]$ & 0.87 & Concomitant MTX therapy \\
\hline Monotherapy & 4 & 18 & & & did not significantly affect \\
\hline MTX $^{a}$ & 5 & 19 & & & LTR outcome \\
\hline $\mathrm{CYA}^{\mathrm{a}}$ & 0 & 2 & & & $\begin{array}{l}\text { Concomitant CYA therapy } \\
\text { was not tested due to } \\
\text { inadequate sample size }\end{array}$ \\
\hline Side effects & & & $0.62[0.13,2.84]$ & 0.54 & There is no significant \\
\hline No & 3 & 17 & & & $\begin{array}{l}\text { difference in likelihood of } \\
\text { achieving LTR between }\end{array}$ \\
\hline Yes & 6 & 21 & & & $\begin{array}{l}\text { patients who reported side } \\
\text { effects and patients who did } \\
\text { not report side effects }\end{array}$ \\
\hline
\end{tabular}

LTR long-term response, $O R$ odds ratio, $C I$ confidence interval, $B M I$ body mass index, $N W$ normal weight, $O W$ overweight, $O B$ obese, non-NW OW + OB, $M T X$ methotrexate, $C Y A$ cyclosporine

*Significant result

${ }^{a}$ One patient used both MTX and CYA 
Table 5 Multiple linear regression

\begin{tabular}{lclcc}
\hline & Estimate & Std. error & $\boldsymbol{T}$ value & $\operatorname{Pr}(>|t|)$ \\
\hline Intercept & 79.049 & 7.304 & 10.822 & 0.0000 \\
Age $(\geq 45$ years $)$ & 1.447 & 3.546 & 0.408 & 0.6855 \\
Gender (M) & 7.132 & 3.472 & 2.054 & $0.0467^{*}$ \\
Non-NW & 12.112 & 4.462 & 2.715 & $0.0098^{*}$ \\
Strength increase & -1.350 & 4.785 & -0.282 & 0.7794 \\
MTX & -1.631 & 3.563 & -0.458 & 0.6498 \\
Side effects (Y) & -0.044 & 3.298 & -0.013 & 0.9895 \\
\hline
\end{tabular}

*Significant

Table 6 Odds ratio and 95\% confidence intervals for inter-variable correlations

\begin{tabular}{|c|c|c|c|c|c|c|}
\hline & Age & Gender & BMI & Strength increase & MTX & Side effects \\
\hline Age & & $0.61[0.19,1.92]$ & $0.95[0.21,4.35]$ & $0.31[0.07,1.39]$ & $2.33[0.73,7.43]$ & $1.02[0.33,3.20]$ \\
\hline Gender & & & $0.63[0.13,3.01]$ & $0.43[0.10,1.91]$ & $1.40[0.45,4.38]$ & $0.81[0.26,2.54]$ \\
\hline BMI & & & & $1.29[0.22,7.65]$ & $1.05[0.23,4.82]$ & $1.29[0.28,5.94]$ \\
\hline Strength increase & & & & & $5.50[1.03,29.45]^{*}$ & $4.00[0.89,18.01]$ \\
\hline MTX & & & & & & $2.36[0.73,7.60]$ \\
\hline Side effects & & & & & & \\
\hline
\end{tabular}

*Significant

The underlying pathophysiology of how obesity may affect long-term response to INF is not well understood. Obese patients may require a dosage increase due to variations in drug clearance and pharmacokinetics [30]. Clinical efficacy has been postulated to relate to INF trough concentration $\left(C_{\min }\right)$. This study by Colls-Gonzalez et al. reported that INF $C_{\min }$ was significantly associated with antibodies to infliximab and BMI [31].

\section{Concomitant Therapy}

In the LTR group, 50\% (18/36) of patients were also taking MTX during their INF treatment, whereas only $22 \%$ of the no LTR group reported concomitant MTX use. CYA was rarely added, with only two patients from the entire population prescribed CYA, including one patient who took both MTX and CYA simultaneously. Monotherapy with INF is typically sufficient to achieve clinical benefit in the average population. However, the patients reviewed in our study were all undergoing a longer than average therapy course. This prolonged treatment course may be due to the lack or loss of response, thus prompting clinicians to prescribe an additional agent such as MTX or CYA.

Given that INF is a chimeric monoclonal antibody with a murine component, immunogenicity with the development of antidrug antibodies (ADA), or specifically antibodies to infliximab (ATIs), is relatively common. These ATIs reduce INF treatment efficacy and potentially increase the risk of infusion reactions. In the pivotal EXPRESS I clinical trial, results show that the presence of ATIs had an effect on maintenance, with only $39 \%$ of patients who 
tested positive for ATIs maintaining PASI 75 response rates at week 50 compared to $81 \%$ of patients without ATIs [14]. The results from the EXPRESS II clinical trial reports $36 \%$ and $51 \%$ of patients receiving continuous INF $5 \mathrm{mg} / \mathrm{kg}$ and $3 \mathrm{mg} / \mathrm{kg}$, respectively, for 1 -year duration were also positive for ATIs. However, the clinical significance of this apparent increased immunogenicity on efficacy and infusion reactions is unknown [20]. A systematic review including ten INF studies (four randomized controlled trials, four prospective, and two retrospective studies) also examined the impact of concomitant methotrexate and the development of ATIs. Though no definitive conclusions can be made based on limited data availability, the addition of methotrexate appears to favor a beneficial effect on reducing or preventing ATIs; however, further studies are needed [32]. In dermatology studies, INF was prescribed as monotherapy, whereas in rheumatology and gastroenterology studies, a significant proportion (>50\%) were maintained on concomitant systemic therapies, including MTX.

The addition of concomitant immunosuppressive therapy with MTX has been shown to augment the efficacy of INF; there are multiple hypotheses suggesting why this may be. First, a number of rheumatologic studies have demonstrated that concomitant MTX reduces the production of ATIs [32-36]. MTX may also lead to a synergistic or additive effect to INF by inducing apoptosis of lamina propria $\mathrm{T}$ lymphocytes and monocytes, a main mechanism of action of INF [37-39]. Studies have also suggested drug interactions between MTX and INF where MTX may reduce the clearance of INF $[36,40]$. Though our results do not show concomitant MTX to be positively associated with LTR, a greater percentage of patients achieving LTR received concomitant MTX compared to patients not achieving LTR (50\% vs. $22 \%$ ).

\section{Side Effects}

The number of patients who experienced side effects was higher in the no LTR group with $67 \%$ reporting at least one side effect. The LTR group had $55 \%$ of patients with side effects.
Statistical analysis did not find this difference to be of significance. Common side effects reported were upper and lower respiratory infections, infusion-site discomfort, fatigue, and changes in mood. The most severe side effects included reactivation of latent infections (zoster and tuberculosis) as well as organ failure.

Based on data from 4779 adult patients exposed (2625 patients beyond 30 weeks and 374 exposed beyond 1 year) to INF (1304 rheumatoid arthritis, 1106 Crohn's disease, 202 ankylosing spondylitis, 293 psoriatic arthritis, 484 ulcerative colitis, 1373 plaque psoriasis, and 17 other conditions), infusion-related reactions (e.g., dyspnea, flushing, headache, and rash) were one of the most common reasons for treatment discontinuation (3\%). In clinical trials, infusion reactions were defined as any adverse event occurring during an infusion or within $1-2 \mathrm{~h}$ post-infusion. Of all patients exposed to INF in the clinical studies, 20\% experienced an infusion reaction compared to approximately $10 \%$ for placebo-treated patients. Serious infusion reactions (i.e., anaphylaxis, seizures, erythematous rash, and hypotension) occurred in $<1 \%$ of patients. Overall, patients who tested positive for ATIs were more likely to experience an infusion reaction, with the use of concomitant immunosuppressants (i.e., methotrexate) likely reducing the frequency of both ATIs and infusion reactions $[12,14,20]$.

\section{Limitations}

Due to the transition from paper charts to electronic medical records (EMR), patients who began therapy prior to the transition were excluded from the study. The difficulty associated with comparing the styles of the paper documentation to the EMR introduced too much variability and was determined to be inadequate and inefficient for this study. One limitation of this study was the use of tBSA as a response outcome. A standardized PASI or Physician Global Assessment (PGA) score that takes into account not only weighted tBSA, but also erythema, induration, and desquamation is a more accurate response variable. For some 
patients, the tBSA was estimated by using the Wallace Rule of Nines and the physical examination description as outlined in the methods, which provided a source for variability and error. Another limitation is the possibility of confounding variables not included in the statistical analysis. This includes age, concomitant medications, prior treatments, or comorbidities. The study may also have been subjected to selection bias as our psoriasis specialty clinic in Dallas is a high-volume psoriasis clinic (over 4000 current patients) where recalcitrant and generalized psoriasis patients come to seek care. Therefore, the sample of psoriasis patients may not be representative of the general psoriasis population, even with moderate-to-severe psoriasis. Finally, the modest sample size $(n=47)$ and long-term therapy ( $\geq 5$ years) may have potentially decreased the statistical power of the results.

\section{CONCLUSIONS}

Contrary to our hypothesis that higher BMI would negatively correlate with LTR, our findings reveal that obesity is positively correlated with achieving a long-term ( $\geq 5$ years) response. This may be related to our methods of statistical analysis, or less likely, to underlying pathophysiologic mechanisms relating to obesity and immunosuppressive state. These conclusions may aid clinicians when making treatment decisions on biologic therapy. These results emphasize the need for future clinical research examining the impact of biosimilars, comorbidities, ATIs, and prior treatments on the long-term response of psoriasis patients to INF as well as to other biologics.

\section{ACKNOWLEDGEMENTS}

We thank the patients for participating in this study and Jinghua $\mathrm{Gu}$ for providing statistical data analysis assistance.
Funding. No funding or sponsorship was received for this study or publication of this article.

Authorship. All named authors meet the International Committee of Medical Journal Editors (ICMJE) criteria for authorship for this article, take responsibility for the integrity of the work as a whole, and have given their approval for this version to be published.

Disclosures. Emily Haque, Aaminah Azhar, John Corbett, Jillian Frieder, and Xuan Wang have nothing to disclose. Alan Menter reports grants and honoraria from Abbott Labs, Amgen Boehringer Ingelheim, Janssen Biotech, LEO Pharma, and SunPharma; grants from Celgene and Merck; and honoraria from Eli-Lilly, Novartis, and UCB, all outside of the submitted work. Alan Menter is also a member of the journal's Editor in Chief.

Compliance with Ethics Guidelines. This retrospective analytic study was approved by the Baylor Scott \& White Research Institute's Institutional Review Board. All procedures performed in studies involving human participants were in accordance with the institutional research committee and with the 1964 Helsinki Declaration and its later amendments or comparable ethical standards. Informed consent was obtained from all individual participants included in the study.

Data Availability. All data generated or analyzed during this study are included in this published article or as supplementary information files.

Open Access. This article is licensed under a Creative Commons Attribution-NonCommercial 4.0 International License, which permits any non-commercial use, sharing, adaptation, distribution and reproduction in any medium or format, as long as you give appropriate credit to the original author(s) and the source, provide a link to the Creative Commons licence, and indicate if changes were made. The images or other third party material in this article are included in the article's Creative Commons 
licence, unless indicated otherwise in a credit line to the material. If material is not included in the article's Creative Commons licence and your intended use is not permitted by statutory regulation or exceeds the permitted use, you will need to obtain permission directly from the copyright holder. To view a copy of this licence, visit http://creativecommons.org/licenses/by$\mathrm{nc} / 4.0 /$.

\section{REFERENCES}

1. Vanderpuye-Orgle J, Zhao Y, Lu J, et al. Evaluating the economic burden of psoriasis in the united states. J Am Acad Dermatol. 2015;72(961):967.e5.

2. Liang SE, Cohen JM, Ho RS. Psoriasis and suicidality: a review of the literature. Dermatol Ther. 2019;32:e12771.

3. Koo J, Marangell LB, Nakamura M, et al. Depression and suicidality in psoriasis: review of the literature including the cytokine theory of depression. J Eur Acad Dermatol Venereol. 2017;31:1999-2009.

4. Menter A, Strober BE, Kaplan DH, et al. Joint AADNPF guidelines of care for the management and treatment of psoriasis with biologics. J Am Acad Dermatol. 2019;80:1029-72.

5. Korman NJ, Zhao Y, Pike J, Roberts J. Relationship between psoriasis severity, clinical symptoms, quality of life and work productivity among patients in the USA. Clin Exp Dermatol. 2016;41: 514-21.

6. Kirby B, Richards HL, Mason DL, Fortune DG, Main CJ, Griffiths CEM. Alcohol consumption and psychological distress in patients with psoriasis. $\mathrm{Br} \mathrm{J}$ Dermatol. 2008;158:138-40.

7. Abuabara K, Azfar RS, Shin DB, Neimann AL, Troxel $\mathrm{AB}$, Gelfand JM. Cause-specific mortality in patients with severe psoriasis: a population-based cohort study in the UK. Br J Dermatol. 2010;163:586-92.

8. Neimann AL, Shin DB, Wang X, Margolis DJ, Troxel $\mathrm{AB}$, Gelfand JM. Prevalence of cardiovascular risk factors in patients with psoriasis. J Am Acad Dermatol. 2006;55:829-35.

9. Najarian DJ, Gottlieb AB. Connections between psoriasis and Crohn's disease. J Am Acad Dermatol. 2003;48:805-24.

10. Menter A, Korman NJ, Elmets CA, et al. Guidelines of care for the management of psoriasis and psoriatic arthritis: Section 6. Guidelines of care for the treatment of psoriasis and psoriatic arthritis: Case-based presentations and evidence-based conclusions. J Am Acad Dermatol. 2011;65:137-74.

11. Carrascosa J, Jacobs I, Petersel D, Strohal R. Biosimilar drugs for psoriasis: principles, present, and near future. Dermatol Ther. 2018;8:173-94.

12. Shear N. INFLIXIMAB. In: Shear N, editor. Litt's drug eruption \& reaction manual 25E. CRC Press. 2019. p. 147-8. https://doi.org/10.1201/97804290 20162.

13. Papp KA, Lebwohl MG. Onset of action of biologics in patients with moderate-to-severe psoriasis. J Drugs Dermatol. 2017;17:247.

14. Reich K, Nestle FO, Papp K, et al. Infliximab induction and maintenance therapy for moderateto-severe psoriasis: a phase III, multicentre, doubleblind trial. Lancet. 2005;366:1367-74.

15. Chaudhari U, Romano P, Mulcahy L, Dooley L, Baker D, Gottlieb A. Efficacy and safety of infliximab monotherapy for plaque-type psoriasis: a randomised trial. Lancet. 2001;357:1842-7.

16. Gottlieb AB, Evans R, Li S, et al. Infliximab induction therapy for patients with severe plaque-type psoriasis: a randomized, double-blind, placebocontrolled trial. J Am Acad Dermatol. 2004;51: 534-42.

17. Gottlieb AB, Masuda S, Ramamurthi R, et al. Pharmacodynamic and pharmacokinetic response to anti-tumor necrosis factor- $\alpha$ monoclonal antibody (infliximab) treatment of moderate to severe psoriasis vulgaris. J Am Acad Dermatol. 2003;48:68-75.

18. Nast A, Sporbeck B, Rosumeck S, et al. Which antipsoriatic drug has the fastest onset of action? Systematic review on the rapidity of the onset of action. J Clin Investig Dermatol. 2013;133:1963-70.

19. Antoni CE, Kavanaugh A, Kirkham B, et al. Sustained benefits of infliximab therapy for dermatologic and articular manifestations of psoriatic arthritis: Results from the infliximab multinational psoriatic arthritis controlled trial (IMPACT). Arthritis Rheum. 2005;52:1227-366.

20. Menter A, Feldman SR, Weinstein GD, et al. A randomized comparison of continuous vs. intermittent infliximab maintenance regimens over 1 year in the treatment of moderate-to-severe plaque psoriasis. J Am Acad Dermatol. 2007;56:31.e1-31. e15.

21. Jacobi A, Langenbruch A, Purwins S, Augustin M, Radtke MA. Prevalence of obesity in patients with 
psoriasis: results of the national study PsoHealth3. Dermatology. 2015;231:231-8.

22. Rodríguez-Cerdeira C, Cordeiro-Rodríguez M, Carnero-Gregorio $\mathrm{M}$, et al. Biomarkers of inflammation in obesity-psoriatic patients. Mediat Inflamm. 2019;2019:7353420.

23. Ottaviani S, Allanore Y, Tubach F, et al. Body mass index influences the response to infliximab in ankylosing spondylitis. Arthritis Res Ther. 2012;14: R115.

24. Singh S, Dulai PS, Zarrinpar A, Ramamoorthy S, Sandborn WJ. Obesity in IBD: epidemiology, pathogenesis, disease course and treatment outcomes. Nat Rev Gastroenterol Hepatol. 2016;14: 110-21.

25. Scaldaferri F, D'Ambrosio D, Holleran G, et al. Body mass index influences infliximab post-infusion levels and correlates with prospective loss of response to the drug in a cohort of inflammatory bowel disease patients under maintenance therapy with infliximab. PLoS ONE. 2017;12:e0186575.

26. Singh S, Facciorusso A, Singh AG, et al. Obesity and response to anti-tumor necrosis factor- $\alpha$ agents in patients with select immune-mediated inflammatory diseases: a systematic review and meta-analysis. PLoS ONE. 2018;13:e0195123.

27. Painter SD, Ovsyannikova IG, Poland GA. The weight of obesity on the human immune response to vaccination. Vaccine. 2015;33:4422-9.

28. Crouch M, Guesdon W, Shaikh S. Obesity suppresses B cell development and impairs antibody production upon antigen challenge. FASEB J. 2017;31(964):7.

29. Højgaard P, Glintborg B, Kristensen LE, Gudbjornsson B, Love TJ, Dreyer L. The influence of obesity on response to tumour necrosis factor- $\alpha$ inhibitors in psoriatic arthritis: results from the DANBIO and ICEBIO registries. Rheumatology (Oxford). 2016;55:2191-9.

30. Puig L. Obesity and psoriasis: Body weight and body mass index influence the response to biological treatment. J Eur Acad Dermatol Venereol. 2011;25: 1007-111.

31. Colls-Gonzalez M, Notario-Rosa J, Bas-Minguet J, et al. Association between infliximab concentrations and clinical response in psoriasis: a prospective cohort study. J Dermatol Treat. 2019. https://doi.org/10.1080/09546634.2019.1690623.

32. Hsu L, Snodgrass BT, Armstrong AW. Antidrug antibodies in psoriasis: a systematic review. Br J Dermatol. 2014;170:261-73.

33. Baert F, Noman M, Vermeire S, et al. Influence of immunogenicity on the long-term efficacy of infliximab in Crohn's disease. $\mathrm{N}$ Engl J Med. 2003;348:601-8.

34. Hanauer SB, Feagan BG, Lichtenstein GR, et al. Maintenance infliximab for Crohn's disease: the ACCENT I randomised trial. Lancet. 2002;359: 1541-9.

35. Hanauer SB, Wagner CL, Bala $\mathrm{M}$, et al. Incidence and importance of antibody responses to infliximab after maintenance or episodic treatment in Crohn's disease. Clin Gastroenterol Hepatol. 2004;2:542-53.

36. Maini RN, Breedveld FC, Kalden JR, et al. Therapeutic efficacy of multiple intravenous infusions of anti-tumor necrosis factor $\alpha$ monoclonal antibody combined with low-dose weekly methotrexate in rheumatoid arthritis. Arthritis Rheum. 1998;41: 1552-633.

37. Van den Brande JMH, Braat $H$, van den Brink GR, et al. Infliximab but not etanercept induces apoptosis in lamina propria T-lymphocytes from patients with Crohn's disease. Gastroenterology. 2003;124:1774-855.

38. Shen C, Maerten P, Geboes K, Van Assche G, Rutgeerts $\mathrm{P}$, Ceuppens JL. Infliximab induces apoptosis of monocytes and $\mathrm{T}$ lymphocytes in a humanmouse chimeric model. Clin Immunol. 2005;115: 250-9.

39. Lügering A, Schmidt $M$, Lügering $N$, Pauels $H$, Domschke W, Kucharzik T. Infliximab induces apoptosis in monocytes from patients with chronic active Crohn's disease by using a caspase-dependent pathway. Gastroenterology. 2001;121: 1145-57.

40. Schwab M, Klotz U. Pharmacokinetic considerations in the treatment of inflammatory bowel disease. Cham: Adis International; 2001. p. 723. 